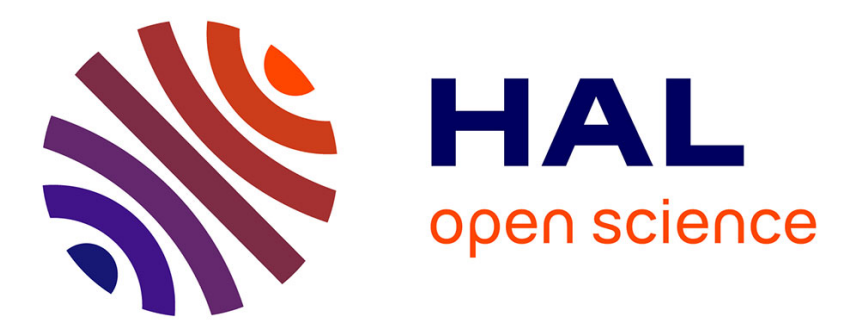

\title{
Optical absorption parameters of amorphous carbon films from Forouhi-Bloomer and Tauc-Lorentz models: a comparative study
}

\author{
N. Laidani, R. Bartali, G. Gottardi, M. Anderle, Pierre Cheyssac
}

\section{- To cite this version:}

N. Laidani, R. Bartali, G. Gottardi, M. Anderle, Pierre Cheyssac. Optical absorption parameters of amorphous carbon films from Forouhi-Bloomer and Tauc-Lorentz models: a comparative study. Journal of Physics: Condensed Matter, 2008, 20 (1), pp.015216.1-015216.8. 10.1088/09538984/20/01/015216. hal-00434409

\author{
HAL Id: hal-00434409 \\ https://hal.science/hal-00434409
}

Submitted on 7 Dec 2009

HAL is a multi-disciplinary open access archive for the deposit and dissemination of scientific research documents, whether they are published or not. The documents may come from teaching and research institutions in France or abroad, or from public or private research centers.
L'archive ouverte pluridisciplinaire HAL, est destinée au dépôt et à la diffusion de documents scientifiques de niveau recherche, publiés ou non, émanant des établissements d'enseignement et de recherche français ou étrangers, des laboratoires publics ou privés. 


\title{
Optical absorption parameters of amorphous carbon films from Forouhi-Bloomer and Tauc-Lorentz models: a comparative study
}

\author{
N. Laidani*, R. Bartali, G. Gottardi, M. Anderle \\ Fondazione Bruno Kessler - Ricerca Scientifica e Tecnologica, Via Sommarive, 18 \\ 38050 Povo (Trento), Italy.
}

\section{P. Cheyssac}

Laboratoire de Physique de la Matière Condensée ( UMR CNRS 6622), Université de Nice Sophia-Antipolis, Parc Valrose,

06108 Nice cedex 2, France.

\begin{abstract}
Parametrization models of optical constants, namely Tauc-Lorentz (TL), Forouhi-Bloomer (FB) and modified FB models, were applied to the interband absorption of amorphous carbon films. The optical constants were determined by means of transmittance and reflectance measurements in the visible range. The studied films were prepared by rf sputtering and characterized for their chemical properties. The analytical models were also applied to other optical data published in literature, pertaining to films produced by various deposition techniques. The different approaches to determine important physical parameters of the interband transition yielded different results. A figure-of-merit was introduced to check the applicability of the models and the results showed that FB modified for an energy-dependence of the dipole matrix element represents adequately the interband transition in the amorphous carbons. Further, the modified FB model shows a relative superiority over TL one's for what concerns the determination of the band gap energy, as it is the only one to be validated by an independent, though indirect, gap measurement by XPS. Finally, the application of the modified FB model allowed to establish some important correlations between film structure and optical absorption properties.
\end{abstract}

PACS:

78.20.-e; 78.20.Ci; 78.30.Ly; 78.30.Am

\footnotetext{
* Corresponding author

Phone : +39-0461314 453

Fax. : +39-0461 810851
} 


\section{1- INTRODUCTION}

Thanks to their many useful properties such as mechanical resistance, chemical inertness, low friction, amorphous carbon films (hydrogenated or un-hydrogenated diamond-like and polymerlike) can cover a broad range of applications. These materials are also attractive candidates as host matrices for incorporation of nanocrystalline metals. These nanocomposites, with carbon-based matrices instead of transparent media like $\mathrm{SiO}_{2}$ or $\mathrm{Al}_{2} \mathrm{O}_{3}$, found interest for applications in photonic devices [1-3]. Such carbon-based nanocomposites exhibit interesting properties like surface plasmon resonance and field emission properties. They are also proposed as solar absorbers materials [4]. However, contrasting with the other dielectrics, amorphous carbons are a class of materials with a wide range of optical and dielectric properties due to a structure formed by atoms with different degrees of electron hybridization and affected by different kinds of disorder, properties tightly correlated with the film synthesis conditions. Consequently, an accurate knowledge of the properties of the carbonaceous matrix is of considerable importance for designing carbon-based nanocomposites with specific performances.

Generally, optical absorption evaluation of amorphous insulators and semi-conductors requires the parametrization of the photon energy dependence of the optical constants (refractive index, extinction coefficient, dielectric function). Several models have been developed to describe the electronic transition parameters such as the band gap, the peak transition energy and the transition lifetime. These models, namely Tauc [5], Tauc-Lorentz (TL) [6] and Forouhi-Bloomer (FB) [7], models, are of wide use. They have been applied to amorphous carbon films too, however few studies were made to check their validity in the case of this particular class of materials [8,9]. It is worthwhile to note that this kind of studies received new interest in recent works for high- $k$ [10], and amorphous chalcogenide [11] and indium nitride films [12] for example.

In this work, hard amorphous carbon films ( $\mathrm{a}-\mathrm{C}$ ) were prepared by $\mathrm{rf}$ sputtering and characterized from structural and optical point of views. Application of TL and FB parametrization models was made to the optical constants, with the introduction of a figure-of-merit based on the equality of the peak transition energy and the maximum absorption energy derived from the models. The results show the adequacy of FB model to describe the interband transition provided an energy dependence of the dipole matrix element of the transition is introduced. The self-consistency of the model is thus achieved not only for the sputtered films of this study but also for other films from literature. Furthermore, the application of the modified FB model allowed to establish some important correlations between film structure and optical absorption properties. 


\section{2- EXPERIMENTAL DETAILS}

\section{1- Film deposition:}

The amorphous carbon films were sputter-deposited from graphite in $5 \mathrm{~Pa}$ r.f $(13.56 \mathrm{MHz})$ discharges in an Ar- $\mathrm{H}_{2}$ mixture containing from 0 to $84 \% \mathrm{H}_{2}$ and with a total gas flux of $30 \mathrm{sccm}$. The samples were mounted on a rotating support, water-cooled to room temperature, and at a distance of $8 \mathrm{~cm}$ from the cathode. In order to obtain different $\mathrm{C} / \mathrm{H}$ compositions and network structures in the films, various energy conditions of plasma were applied either through the feed gas composition variation when a plasma was created with a continuous rf wave $(\mathrm{CW})$ [13], or through the modulation of the RF discharge for a given feedgas composition [14]. The rf discharge modulation was achieved by pulsing with 1-100 $\mathrm{kHz}$ frequencies. For the $\mathrm{CW}$ plasmas, a constant - $550 \mathrm{~V}$ dc self-bias of the cathode was maintained. The modulated discharges were also produced with $-550 \mathrm{~V}$ discharge voltage either during the "time on" of the pulse with a $0 \mathrm{~V}$ "time off" value, or as an average over the "on" and "off" times of the pulse.

As the present work focuses only on different parametrization models applicability for optical constants of a wide set of carbon films, no weight is directly given to the process parameters effects themselves on the film structure. More details of such study can be found in [13-15].

Three kinds of substrates were used: $175 \mu \mathrm{m}$ thick polyethylene terephtalate (PET) sheets and 1.02 $\mathrm{mm}$ thick polycarbonate sheets for optical analysis, both supplied by Goodfellow and n-type $\operatorname{Si}(100)$ wafers for chemical analysis. Series of samples were prepared with various film thicknesses, ranging from $\sim 50 \mathrm{~nm}$ to $\sim 500 \mathrm{~nm}$.

\section{2- Film characterization}

X-ray photoelectron spectroscopy (XPS) spectra were recorded with a Scienta ESCA 200 spectrometer, and the monochromatized $\mathrm{AlK} \alpha(1486.6 \mathrm{eV})$ radiation. Survey and detailed scans spectra were recorded and after a Shirley-type background subtraction, raw spectra were fitted using a non-linear fitting program adopting Gaussian-Lorentzian peak shape. Instead, for the valence band spectra, a linear background subtraction was applied. A highly-oriented pyrolytic graphite (HOPG) standard sample as well as a standard diamond film [16] were also analysed.

Optical absorption measurements were made with a Jasco V-550 spectrophotometer in the 400$800 \mathrm{~nm}$ wavelength range. Reflectance spectra were recorded in the visible range, at normal incidence. An Al mirror was used as a $100 \%$ reflectance reference material. Before measuring the 
sample reflectance, a second $\mathrm{Al}$ mirror was measured with respect to the first one in order to assess the experimental measurement error, which was found as $0.5 \%$. Transmittance spectra of samples have also been measured taking the bare substrate as reference.

Fourier-transform infra-red spectroscopy (FT-IR) was used to quantify the chemically bound hydrogen content of the films. It was carried out with a Bio-Rad FTS 185 spectrometer equipped with a DTGS detector and a KBr beam splitter, in the absorbance mode and within the 400-4000 $\mathrm{cm}^{-1}$ wavenumber range. In particular the $2600-3200 \mathrm{~cm}^{-1}$ interval was considered. It corresponds to the stretching vibration modes of various $\mathrm{C}-\mathrm{H}$ bonds and allows to determine the composition of the hydrogenated phase of the film structure.

\section{3- RESULTS}

\section{1- Background}

Optical absorption measurements are widely used to characterize the electronic properties of materials, through the determination of parameters describing the electronic transitions such as: band gap, valence band tails and lifetime of excited state, which can be related to disorder in the material network i.e. bond strength and defects. However, the optical absorption depends not only on a convolution of the density of states of conduction and valence bands (CB and VB), but also on the matrix elements of electronic transitions. From optical properties alone, it is not possible to uniquely determine electronic properties without simplifying assumptions about these matrix elements and the density of states.

From the quantum mechanics of optical absorption, within the one-electron approximation, it is shown that the imaginary part $\varepsilon_{2}(\mathrm{E})$ of the relative dielectric function is

$$
\varepsilon_{2}(E)=\left(\frac{2 \pi e \hbar}{m}\right)^{2}\left(\frac{2}{V E^{2}}\right) \sum\left|P_{v, c}\right|^{2} \delta\left(E_{c}-E_{v}-E\right)
$$

where $\mathrm{E}$ is the energy of the incident light wave, $\mathrm{V}$ is the illuminated volume, e and $\mathrm{m}$ the electron charge and mass respectively, $E_{v}$ the energy of the initial (valence) state and $E_{c}$ that of the final (conduction) state. $\left|P_{v, c}\right|^{2}$ is the square of the momentum matrix element associated with the transition. (Note that we use in this work indifferently the terms "initial" and "valence" as well as "final " and "conduction" for the states involved in the transition.)

Equation (1) can be equivalently rewritten in terms of the squared dipole matrix element, $\left|R_{v, c}\right|^{2}$, as 
$\varepsilon_{2}(E)=(2 \pi e)^{2}\left(\frac{2}{V}\right) \sum\left|R_{v, c}\right|^{2} \delta\left(E_{c}-E_{v}-E\right)$

Several models are commonly used to determine the optical properties of amorphous semiconductors and dielectrics in the energy range of interband transitions. The most widespread is Tauc model [5] which allows to derive the band gap energy Eg from $E \sqrt{\varepsilon_{2}}$ as a function of the incident energy E. Eg is then obtained by extrapolating $E \sqrt{\varepsilon_{2}}$ to zero, according to $E \sqrt{\varepsilon_{2}}=B_{T}\left(E-E_{g}\right)$. Tauc's approximation considers a parabolic behaviour of the bands near the edge; $\mathrm{B}_{\mathrm{T}}$ is a constant and the square of the average matrix element of the dipolar momentum

$$
\mathrm{P}^{2}(\mathrm{E})=\left|P_{v, c}\right|^{2}=|<c| p|v\rangle^{2} \mid
$$

is assumed to be independent of the photon energy $\mathrm{E}$.

In the Tauc-Lorentz model, introduced by Jellison and Modine [6], $\varepsilon_{2}(\mathrm{E})$ is given as the product of Tauc function: $G(E) \alpha \frac{E-E_{g}}{E^{2}}$ by the Lorentz oscillator function $\mathrm{L}(\mathrm{E})$ :

$\mathrm{L}(\mathrm{E})=\mathrm{AE}_{0} \Gamma \mathrm{E} /\left[\left(\mathrm{E}^{2}-\mathrm{E}_{0}^{2}\right)+\Gamma^{2} \mathrm{E}^{2}\right]$

Hence $\varepsilon_{2}(E)=\frac{1}{E}\left[\frac{A E_{0} \Gamma\left(E-E_{g}\right)^{2}}{\left[E^{2}-E_{0}{ }^{2}\right)^{2}+\Gamma^{2} E^{2}}\right]$ for $\mathrm{E}>\mathrm{E}_{\mathrm{g}}$

and $\varepsilon_{2}(E)=0$ for $\mathrm{E} \leq \mathrm{E}_{\mathrm{g}}$.

A is the amplitude factor, proportional to the density of the material and to the momentum matrix element. $E_{0}$ is the peak transition energy that corresponds to the so-called Penn gap. $\Gamma$ is the broadening parameter, inversely related to short-range order [17] and crystallite size [18].

Similarly to TL model, in Forouhi and Bloomer's formalism, important parameters such as the transition lifetime $\tau$, the energy gap between the top of the valence band and the bottom of the conduction band and the peak transition energy can be derived [7, 19]. This is achieved by fitting the extinction coefficient, i.e. the imaginary part $\kappa(E)$ of the refractive index with

$\kappa(\mathrm{E})=\mathrm{A}(\mathrm{E}-\mathrm{Eg})^{2} /\left(\mathrm{E}^{2}-\mathrm{BE}+\mathrm{C}\right)$

where $\mathrm{A}$, is related to the transition dipole matrix element as

$A \propto \frac{1}{\tau}|<c| x|v>|^{2}$

$\mathrm{B}=2\left(\mathrm{E}_{\mathrm{c}}-\mathrm{E}_{\mathrm{v}}\right)$

and $\mathrm{C}=\left(\mathrm{E}_{\mathrm{c}}-\mathrm{E}_{\mathrm{v}}\right)^{2}+\hbar^{2} / 4 \tau^{2}$.

Here $\left(E_{c}-E_{v}\right)=B / 2$ corresponds to the maximum absorption (or Penn gap, as in the case of $E_{0}$ in the TL model ). 
Although both TL and FB models are based on the assumption of parabolic VB and CB band edges, they contrast between each other for the assumption of the matrix element energy dependency: in FB model, the squared dipole matrix element is considered as constant with energy variation,

$$
\mathrm{R}^{2}(\mathrm{E})=|<c| x\left|v>^{2}\right|
$$

where " $\mathrm{x}$ " is for position.

A same limitation is recalled for both FB and TL models: a non-zero absorption in amorphous semi-conductors at energies below the energy gap. Actually, when $\mathrm{E}<\mathrm{E}_{\mathrm{g}}$, there exists a tail called Urbach' s, due to disorder of the amorphous network [20,21]; this sub-gap energy range will not be considered in the present study.

Knowing the relation between $\mathrm{P}^{2}(\mathrm{E})$ and $\mathrm{R}^{2}(\mathrm{E})$, given by

$$
P^{2}(E)=\frac{m^{2}}{\hbar^{4}} E^{2} R^{2}(E)
$$

it becomes important to know which approximation of an energy-independent matrix element holds for a given material. In this work, we have studied the applicability of existing models for the optical transitions in amorphous carbon films, with the introduction of a figure-of-merit merit that allows checking the model adequacy, with a further validation of the electron properties through an independent charaterization by a non-optical method.

\section{3- Calculation of optical constants from transmittance and reflectance spectra}

Most of the studied films were absorbing enough to give transmittance spectra without interference patterns characteristic of multiple reflections at the various interfaces in the film+substrate system. For such films, the imaginary part $\kappa$ of the complex index $v+i \kappa$ was calculated using $\kappa=\alpha \lambda / 4 \pi$, the absorption coefficient $\alpha$ being given by

$\alpha=\frac{1}{t} \operatorname{Ln}\left(\frac{1-R}{T}\right)$

$\lambda$ is the wavelength, $t$ the film thickness, $\mathrm{T}$ and $\mathrm{R}$ the transmittance and reflectance respectively. At normal incidence in air, the reflectance $\mathrm{R}$ of an absorbing medium of refraction index $v+\mathrm{i} \kappa$ is given by $R=\frac{(v-1)^{2}+\kappa^{2}}{(v+1)^{2}+\kappa^{2}}$; then the real part $v$ of the index was extracted from its values. In Fig. 1(a) two examples of absorption coefficients of films deposited in two kinds of plasmas: one of pure $\mathrm{Ar}$ and the other of an $\operatorname{Ar}(84 \%)-\mathrm{H}_{2}(16 \%)$ mixture, are given as a function of wavelength. 
The reflectance spectra of some samples showed interference patterns in a narrow interval corresponding to the highest wavelengths. In the remaining range of wavelengths, $v$ and $\kappa$ have been determined using the procedure described above.

Few films, though giving transmittance spectra without evident interferences, still presented such patterns in their reflectance spectra over all the wavelength range. A typical spectrum is presented in Fig. 1(b). In such case we proceeded as follows: two series of reflectance spectra were measured: one with the rear face of the substrate kept bare as for all samples described above, and the other with the rear face of the substrate coated by a black absorbing layer of graphite. The purpose of the latter procedure was to minimize the reflection due to the rear face of the rather thick polycarbonate sheet. For such a configuration, the optical constants were then calculated more easily by fitting the reflectance spectrum. Here we give the procedure adopted for a $211 \mathrm{~nm}$ thick film deposited on a polycarbonate substrate, a structure sketched in Fig. 1(c).

The amplitude of the electric field reflected by the sample is described according to the classical principles of propagation of electromagnetic waves, discussed by Born and Wolf [22]: namely we consider a plane and parallel layer of carbon deposited on a semi-infinite substrate of polycarbonate and so we define three interfaces: (1) air/film, (2) film/substrate and (3) substrate/air (Fig. 1(c)). Since the rear face of polycarbonate is coated and absorbing, no wave is back reflected; the combination of interfaces becomes rather straightforward although complex values of indices have to be handled with care.

Let $n_{i}$ be the index of the i-th layer. The reflectance coefficients $\rho_{i j}$ of the various interfaces are $\rho_{i j}=\frac{n_{i}-n_{j}}{n_{i}+n_{j}}$ with $\rho_{12}$ for the vacuum-carbon layer, and $\rho_{23}$ for carbon-polycarbonate. Then, the theoretical expression of Fresnel reflectance is $R$ Fresnel $=\rho \rho^{*}$ with $\rho=\frac{\rho_{12}+\rho_{23} e^{2 i k_{2} d_{2}}}{1+\rho_{12} \rho_{23} e^{2 i k_{2} d_{2}}}$

where $k_{2}$ is the wave vector and $d_{2}$ the thickness of the carbon layer [23]. Inputs of the calculations are indices of polycarbonate $n_{p}=v_{p}+\mathrm{i} \kappa_{p}$, optical index $n=v+\mathrm{i} \kappa$ of carbon and its measured thickness $d_{2}=211 \mathrm{~nm}$.

The indices of polycarbonate were derived from a fit of its reflectance curve. Initial guess values for $v$ and $\kappa$ of carbon were necessary for the calculations. According ref. [24], diamond's Sellmeier dependence of $v$ with $\lambda$ was chosen. The wavelength dependence of $\kappa$ was derived using eq. 10 from $\mathrm{T}$ and $\mathrm{R}$ spectra measured on samples whose substrate was left with a bare rear face. We considered this dispersion as the most realistic one for input values of $\kappa$ in the calculations. As a result, the fit 
of $\mathrm{R}$ is quite good over most of the energy range; as it can be seen in Fig.1(b). The wavelength dispersion parameters of $v(\lambda)$ and $\kappa(\lambda)$, as obtained from the fit of experimental $R(\lambda)$, are given in the inset of Fig. 1(b).

In the visible range, the optical properties of all carbon films studied here are then described by the real and imaginary parts of either their index $n=v+i \kappa$ or their dielectric function $\varepsilon=\varepsilon_{1}+\mathrm{i} \varepsilon_{2}$.

\section{4- FB model self-consistency checking and consequent model modification}

The values of optical constants that have been obtained were used to study the absorption properties i.e. the parameters of electronic transitions of the amorphous carbon films.

$\kappa($ E) of all samples has been fitted to FB model which allowed to quantify the transition parameters, in particular the values of the optical gap $\left(E_{g}\right)$ and the peak transition $(B / 2)$ which will be discussed.

From the parameters of FB fit, we also derived the energy $E_{\max }$, corresponding to the maximum of absorption (maximum of $\kappa$ ). $\mathrm{E}_{\max }$ and $\mathrm{B} / 2$ should be very close as they have the same physical significance. So, for checking the model applicability, we took at first, the equality of $B / 2$ and $E_{\max }$ values as a figure-of-merit. The two parameters are plotted in Fig.2(a) which shows clearly the lack of correspondence between them, evidenced by the linear fit of the data which gives a negative slope. Furthermore, we found that this inconsistency was not only restricted to carbon films only, but the latter are among amorphous materials for which FB parametrization is the least applicable. In order to illustrate these remarks, we selected a wide range of amorphous materials and, also for completeness, crystalline materials for which FB fitting parameters of $\kappa(E)$ were available in a tabulated form in literature. The amorphous solids concerned by such available data are a-C:H [25], tetrahedral a-C [26, 27], nanocrystalline-C:S [28], a-Si, a-Si:H, a-Si $\mathrm{N}_{4}, \mathrm{a}_{-} \mathrm{TiO}_{2}$ [7]. Tested crystalline materials were c-GaAs, c-Si, c- $\mathrm{SiO}_{2}$, diamond [19] and c- $\mathrm{TiO}_{2}$ [29]. In these cases, all possible transitions were considered.

In Fig. 2(b) a semi-log graph of $E_{\max }$ versus $B / 2$ is displayed. We can see that the crystalline solids rigorously follow a one-to-one relationship between the two energies, represented by the solid line in the plot, contrarily to the amorphous ones which exhibit strong deviations, the most drastic ones being for the amorphous carbon films, as introduced before.

The approximation of an energy-independent dipole matrix element does neither make sense when applied to amorphous carbons (Fig.2(a and b), nor to nanocrystalline carbon (Fig. 2(b)).

In order to make FB model tractable with our studied a-C films, we tried another approach that introduces an energy dependence in the squared matrix element $\mathrm{R}^{2}(\mathrm{E})$ of the transition dipole which 
enters the physical model of FB. The assumption of parabolic band edges has been kept, the subgap energy range has been ignored. Our choice of the energy dependence of $R^{2}(E)$ is based on earlier indications of $\mathrm{a} \mathrm{E}^{-2}$ energy scaling of matrix elements in disordered materials [20, 30]. We then introduced as follows this energy dependence $R^{2}(E) \propto E^{-2}$ in FB parametrization:

$$
\kappa(E)=\frac{1}{E^{2}} \frac{A^{*}\left(E-E_{g}\right)^{2}}{E^{2}-B E+C}
$$

Using this formulation, the best fit of experimental $\kappa(E)$ with this formulation gave the $B / 2$ and $E_{\max }$ values which are plotted in Fig.2(c) and linearly fitted with a slope of 0.94 . The equality of B/2 and $\mathrm{E}_{\max }$ chosen as a figure-of-merit is now fairly achieved.

The modified FB formulation (hereafter $\mathrm{mFB}$ ) for $\kappa(\mathrm{E})$ versus photon energy has been applied to a series of amorphous hydrogenated carbon films whose optical constants $\nu$ and $\kappa$ are compiled in [25]. From tabulated values of three films, we plotted $\kappa(E)$ and checked, through the $B / 2=E_{\max }$ figure-of-merit, the validity of the original model and our modified FB one's (square symbols in Fig. 2(b) and in Fig 2(c) respectively). As in the case of our films, it is demonstrated that the mFB model is able to describe the absorption properties of such materials.

\section{5- Validation of mFB model for amorphous carbons}

In order to further validate the $\mathrm{mFB}$ model applicability to amorphous carbons, we compared two parameters: the value of the optical gap Eg resulting from $\kappa(E)$ fit and the valence band maximum (VBM) energy measured by XPS. The latter parameter was chosen as an indirect measure of the band gap since we found a strong correlation between these two energies for selected standard carbon-based materials: diamond ( i.e. a $100 \% \mathrm{sp}^{3}$ hybridised carbon), graphite (a $100 \% \mathrm{sp}^{2}$ hybridised carbon), polyethylene (a 100\% $\mathrm{sp}^{3}$ hybridised hydrogenated carbon with 66.6 at.\% $\mathrm{H}$ ) and para-quaterphenyl which is a polyphenylene oligomer (a $100 \% \mathrm{sp}^{2}$ hybridised hydrogenated carbon, with 42.8 at.\% H). A third polymer, poly(cis-isoprene), which is a mixed $\mathrm{sp}^{3}-\mathrm{sp}^{2}$ hydrogenated carbon (60\%-40\% respectively) and contains 61.5 at. $\% \mathrm{H}$, was also considered. In the present study, XPS spectra from diamond and graphite were acquired from standard materials, whereas those belonging to the three polymers were taken from literature [31]. The VB top energy is defined as the energy of the zero-intensity point of the spectrum at the low binding energy side. The latter is obtained by a linear extrapolation of the region of the spectrum of lowest binding energy. In XPS, all spectra, and in particular for this study the VB and C1s core level ones, were measured with respect to the Fermi level. In order to eliminate any possible effect of a change of 
Fermi level within the gap, due to different structures and/or different levels of hydrogenation of the films, we used the binding energies of $\mathrm{VB}$ maximum $\left(\mathrm{E}_{\mathrm{VBM}}\right)$ and $\mathrm{C} 1 \mathrm{~s}$ core level $\left(\mathrm{E}_{\mathrm{C} 1 \mathrm{~s}}\right)$ and took into consideration their difference, $\Delta \mathrm{E}=\mathrm{E}_{\mathrm{C} 1 \mathrm{~s}}-\mathrm{E}_{\mathrm{VBM}}$. Typical spectra are displayed in Fig. 3(a and b). $\mathrm{E}_{\mathrm{C} 1 \mathrm{~s}}$ is taken as the binding energy of the main peak obtained after deconvolution of $\mathrm{C} 1 \mathrm{~s}$ spectrum into individual peaks for the carbon and diamond films and for graphite. For the spectra taken from literature, $\mathrm{E}_{\mathrm{C} 1 \mathrm{~s}}$ was simply the energy of the $\mathrm{C} 1 \mathrm{~s}$ peak maximum.

The energy difference $\Delta \mathrm{E}$ should reflect more precisely the red shift of the VB maximum in the case of a gap opening. This was found to be valid for both groups of standard materials, hydrogenated and un-hydrogenated carbons. The lower $\Delta \mathrm{E}$ is, the higher $\mathrm{Eg}$, as it is seen in Fig. 3(c). Optical gaps of reference carbons were taken from literature [32-35].

In Fig. 3(d), we report experimental data from optical and XPS measurements on a set of carbon films deposited with various frequency conditions of plasma pulsing in 84 at.\% $\mathrm{Ar}-16$ at.\% $\mathrm{H}_{2}$ [14]. One sees that the values of $\mathrm{E}_{\mathrm{g}}{ }^{\mathrm{mFB}}$ deduced from $\mathrm{mFB}$ model fit well this correlation. The same cannot be said for the original FB model: no such correlation between $\mathrm{E}_{\mathrm{g}}{ }^{\mathrm{FB}}$ and $\Delta \mathrm{E}$ is seen in Fig.3(e) plot.

\section{6- Comparison between the results of $\mathrm{mFB}$ and TL fits}

For all studied films, $\varepsilon_{2}$ (E) has also been fitted to TL model in order to extract $\mathrm{E}_{\mathrm{g}}{ }^{\mathrm{TL}}$ and $\mathrm{E}_{0}{ }^{\mathrm{TL}}$, the energies of the optical gap and maximum absorption, as well as the transition lifetime and to compare them with their corresponding values in $\mathrm{mFB}$ formulation. Fig. 4(a) shows the mutual correspondence between $\mathrm{E}_{0}{ }^{\mathrm{TL}}$ and $\mathrm{E}_{\max }{ }^{\mathrm{mFB}}$. No fair matching of a one-to-one relationship (represented by a straight line in the figure) is observed between these two parameters. In most cases the values deduced from TL model are larger than those from $\mathrm{mFB}$ one; this despite the $\mathrm{E}^{-2}$ scaling of $\mathrm{R}^{2}(\mathrm{E})$ in $\mathrm{mFB}$ model, which turns out as in TL model: a squared constant momentum matrix element (see eq. 9). Furthermore, TL model is much less well validated than the $\mathrm{mFB}$ one by the results of XPS measurements. In Fig. 3 (e), the plot of $\Delta \mathrm{E}=\mathrm{E}_{\mathrm{C} 1 \mathrm{~s}}-\mathrm{E}_{\mathrm{VBM}}$ versus $\mathrm{E}_{\mathrm{g}}^{\mathrm{TL}}$, as defined in the above section, didn't give any similar trend to what already described above. At this stage, this shows a relative superiority of $\mathrm{mFB}$ model to give the energy of the optical gap of these amorphous carbons.

The transition lifetime from $\mathrm{mFB}$ was derived according to Eq. 7 from $\mathrm{C}$ value given by $\kappa(\mathrm{E})$ fit, and that from TL model was calculated as $\mathrm{h} / \Gamma, \Gamma$ coming from $\varepsilon_{2}$ (E) fit according to Eq. 3(a). Here, in most cases transition lifetimes from TL model came to be higher than those from mFB one, by up to a factor 10, as shown in Fig. 4(b). 


\section{8 - Correlations between chemical structure and optical transition parameters of films:}

The optical properties of amorphous $C$ films are known to be dominated by $\pi-\pi^{*}$ and $\sigma-\sigma^{*}$ electronic transitions, along with $\pi-\sigma *$ and $\sigma-\pi *$ transitions $[35,36]$. The $\pi-\pi^{*}$ contribution originates exclusively from $\mathrm{sp}^{2}$ carbon.

In graphite, $\pi-\pi^{*}$ transition is located at $\sim 4 \mathrm{eV}$, whereas the $\sigma-\sigma *$ transitions occur at $\sim 14 \mathrm{eV}$, at a higher energy than in diamond $(\sim 11 \mathrm{eV})$. All these values of energy correspond to the maximum of absorption for a specific interband transition and are not the value of the optical gap which is null for graphite. Moreover, they can consistently be lower in the amorphous materials than in the bulk crystalline counterparts (graphite and diamond in the case of unhydrogenated carbon) [36, 37]. Similarly, the optical gap is used to characterize different kinds of disorder in the structure of amorphous carbon films. It has been shown that the optical gap is controlled by the amount of $\mathrm{sp}^{2}$ sites in the structure but the manner in which this control occurs is still an open subject. A same $\mathrm{sp}^{2}$ $\mathrm{C}$ fraction can result in different optical properties, depending on the local $\mathrm{sp}^{2}$ configuration. In fact, the existing propositions about the effect of $\mathrm{sp}^{2} \mathrm{C}$ on the gap value are oriented to explain the origin of a "gap opening", that is the optical properties of the films are evaluated referring to a perfectly ordered $\mathrm{sp}^{2}$ structure, which would behave like a zero-gap material as graphite. The "gap opening" could therefore be due to the presence of $\mathrm{sp}^{2} \mathrm{C}$ clusters of nanometric size, viewed as graphitic units, thus due to a size effect which would manifest in a blue shift of the optical gap. Alternatively, as described by the revised structural model of Robertson, the clusters would consist of rings and chains bonding configuration rather than condensed aromatic nuclei in "graphitic units" [35].

Let's recall that the $\mathrm{sp}^{2} \mathrm{C}$ phase is the one susceptible to give rise to absorption in the visible energy range. The mFB model, yielding a more precise knowledge of peak transition and optical gap energies, allows to establish correlations between these important parameters and the structure of the materials. In the present case we investigated the effect of the chemical structure, as determined by FT-IR analysis of the same series of films used to validate the model by XPS. In particular, we focused on the $\mathrm{C}-\mathrm{H}_{\mathrm{x}}$ stretching vibrational band. A typical spectrum is presented in Fig.5(a), with its deconvolution into Gaussian individual peaks identified according to published data [38], as shown in the inset of the figure. This way, the hydrogenated $\mathrm{sp}^{2} \mathrm{C}$ phase was found to be due to both aromatic and olefinic carbon, i.e. carbon atoms organised in rings and in chains respectively, in proportions varying with the film deposition process parameters. 
A strong effect of the composition of the hydrogenated $\mathrm{sp}^{2}$ carbon phase on the film optical gap

and peak transition energies was found. Plotting $\mathrm{E}_{\mathrm{g}}{ }^{\mathrm{mFB}}$ and $\mathrm{E}_{\max }{ }^{\mathrm{mFB}}$ versus the ratio of $\mathrm{sp}^{2}$ ring - to $\mathrm{sp}^{2}$ chain band areas, i. e. the area of aromatic $\mathrm{sp}^{2} \mathrm{CH}$ band, labelled "9" in Fig.5(a), ratioed to the areas sum of olefinic $\mathrm{sp}^{2} \mathrm{CH}_{2}+\mathrm{sp}^{2} \mathrm{CH}$ bands ( peaks " 8 " and "7"), puts clearly in evidence the role of the chain bonds in the gap opening and the red shift of the absorption peak. This is illustrated in Fig. 5(b): the higher the chain bonds proportion (the lower the ring one), the larger the gap, $\mathrm{E}_{\mathrm{g}}^{\mathrm{mFB}}$, and the lower the energy $E_{\max }{ }^{m F B}$ of peak transition, meaning thereby a narrowing of the valence band.

\section{CONCLUSION}

In this work, the optical properties of amorphous carbon films have been studied. The goal was to define the parameters of important interband absorption such as band gap and peak transition energies and transition lifetime. Tauc-Lorentz's and Forouhi-Bloomer's commonly used analytical models were applied to the film optical constants. The inconsistency of FB model when applied to amorphous carbons was evidenced. A modification of FB model by introducing an energy dependence of the dipole matrix element (as $\propto \mathrm{E}^{-2}$ ) results as more adequate to describe the interband transition in a-C and a-C:H films, whereas the original FB model was found well-suited for many other amorphous non-carbonaceous semi-conductors and insulators. Furthermore, the modified FB model shows a relative superiority over the TL one for what concerns the determination of the band gap energy, as it is the only one to be validated by measurements by XPS of the valence band edge energy itself related to the bandgap.. Finally, the application of the modified FB model allowed establishing some important correlations between the film structure and its properties of optical absorption.

\section{ACKNOWLEDGMENTS}

This work was supported by Autonomous Province of Trento (Fondo Progetti Ricerca) under the project MicroCombi. Many thanks to V. Micheli for technical assistance.

\section{REFERENCES}

[1] S. Hussain, A. K. Pal, Appl. Surf. Sci. 253 (2007) 3469

[2] S. Hussain, R. K. Roy, A. K. Pal, J. Phys. D: Appl. Phys. 38 (2005) 900

[3] S. Kundoo, P. Saha, K. K. Chattopadhyay, J. Vac. Sci. technol. B 22 (6) (2004) 2709 
[4] A. Schuler, J. Geng, P. Oelhafen, S. Brunold, P. Gantenhein, U. Frei, Solar Energy Mater. \& Solar Cells 60 (2000) 295

[5] J. Tauc, R. Grigorovici, A. Vancu, Phys. Status Solidi 15 (1966) 627

[6] G. E. Jellison, Jr and F. A. Modine, Appl. Phys. Lett. 69 (3) (1996) 371

[7] A. R. Forouhi and I. B.loomer, Phys. Rev. B 34 (10) (1986) 7018

[8] W. A. Mc Gahan, T. Makovicka, J. Hale, J. A. Woollam, Thin Solid Films 253 (1994) 57

[9] T. Yamaguchi, Y. Kaneko, A. H. Jayatissa, M. Aoyama, Thin Solid Films 279 (1996) 174

[10] J. Price, P. Y. Hung, T. Rhoad, B. Foran, A. C. Diebold, Appl. Phys. Lett. 85 (10) (2004) 1701

[11] I. Ohlidal, D. Franta, M. Siler, F. Vizd'a, M. Frumar, J. Jedelsky, J. Omasta, J. Non-Cryst. Solids 352 (2006) 5633

[12] J. M. Khoshman, M. E. Kordesch, J. Non-Cryst. Solids 352 (2006) 5572

[13] N. Laidani, R. Bartali, P. Tosi, M. Anderle, J. Phys. D.:Appl. Phys. 37 (2004) 2593.

[14] N. Laidani, R. Bartali, M. Anderle, P. Chiggiato, G. Chuste, Diamond \& Related Mater. 14 (2005) 1023.

[15] L. Calliari, M. Filippi, N. Laidani M. Anderle, J. Electron Spectrosc., 150 (2006) 40

[16] M. Filippi, L. Calliari, G. Pucella, G. Verona-Rinati, Surf. Sci. 573 (2004) 225

[17] L. Pereira, H. Āguas, E. Fortunato, R. Martins, Appl. Surf. Sci. 253 ( 2006) 339 and references therein.

[18] G. F. Feng, R. Zallen, Phys. Rev. B 40 (1989) 1064

[19] A. R. Forouhi and I. B.loomer, Phys. Rev. B 38 (3) (1988) 1865

[20] G. A. N. Connel, "Optical properties in amorphous semiconductors", Springer Topics in Applied Physics, Amorphous Semiconductors, vol 36 (Ed. M. H. Brosky, Berlin: Springer, 1979) pp 73-111.

[21] C. R. Miranda, A. Antonelli, A. J. R. da Silva, A. Fazzio, J. Non-Cryst. Solids 338-340 (2004) 400

[22] M. Born, E. Wolf, " Principles of Optics", ( $7^{\text {th }}$ ed., Cambridge University Press, Cambridge, MA, 1999)

[23] P. Cheyssac, Optics Comm. 268 (2006) 273.

[24] G. Ghosh , "Handbook of thermo-optics coefficients of optical materials with applications."

(Academic Press, 1998) pp. 5-110

[25] S. A. Alterovitz, N. Savvides, F. W. Smith, J. A. Woollam, in "Handbook of Optical Constants of Solids" (Ed. A. D. Palik, Academic Press, New York, 1998) pp. 837-852.

[26] S. Xu, L. K. Cheah and B. K. Tay, Thin Solid Films 312 (1998) 160 
[27] A. Canillas, M. C. Polo, J. L. Abdujar, J. Sancho, S. Boch, J. Robertson, W. I. Milne, Diamond \& Related Mater. 10 (2001) 1132

[28] S. Gupta, B. R. Weiner, G. Morell, Thin Solid Films 455-456 (1998) 422.

[29] P.Chryscopoulos, D. Davazoglu, C. Trapalis, G. Kordas, Thin Solid Films 323 ( 1998) 188

[30] G. Fanchini, A. Tagliaferro, Diamond and Related Mater. 10 (2001) 191 and references therein

[31] J. F. Moulder, W. F. Stickle, P. E. Sobol, K. D. Bomber, “ Handbook of X-ray Photoelectron Spectroscopy”, ( J. Chastaing, R. C. King Jr., Eds., Physical Electronics, Inc. Eden Prairie, 1995)

[32] T. Tanaka, J. Appl. Phys. 44 (5)1973) 2430

[33] J. L. Bredas, B. Themans, J. G. Fripiat, J. M. André, R. R. Chance, Phys. Rev. B 29 (12) (1984) 6761

[34] S. Najidha, N. S. Saxena, R. Sreeja, C. H. Unnithan, P. Predeep, Mater. Lett. 59 (2005) 3431

[35] J. Robertson, Mater. Sci. Eng. R37 (2002) 129.

[36] S. Logothetidis, Diamond \& Related Mater. 12 (2003) 141

[37] P. K. Giri, , S. Tripurasundari, G. Raghavan, B. K. Panigrahi, P. Magudapathy, K. G. M. Nair, A. K. Tyagi, J. Appl. Phys. 90 (2) (2001) 659

[38] N. B. Colthup, L. H. Daly, S. E. Wiberley, "Introduction to Infrared and Raman Spectrosopies”, Academic Press (Ed.), New York, 1969. 


\section{FIGURE CAPTIONS}

Figure 1: (a) Examples of absorption coefficients of films deposited in Ar and Ar-H2 (84-16)\% plasmas as a function of wavelength

(b) Experimental and calculated reflectance spectra of a-C film on polycarbonate in the case of multiple reflections

(c) Schematic multilayered structure of the a-C / polycarbonate system

Figure 2: (a) $E_{\max }$ versus the energy difference $B / 2$ between the final and initial states of a transition, both parameters determined from the unmodified FB model applied to our carbon films

(b) semi-log graph of $\mathrm{E}_{\max }$ versus $\mathrm{B} / 2$, from $\mathrm{FB}$ model, both parameters determined from tabulated data. The one-to-one relationship is represented by a solid line.

(c) $\mathrm{E}_{\max }$ versus $\mathrm{B} / 2$, both parameters determined from the modified $\mathrm{FB}(\mathrm{mFB})$ model applied to our carbon films (star symbols) and to some literature data [33] (square symbols). The latter are represented with the same symbol in plot (b).

Figure 3: (a) Typical XPS VB spectrum of a-C film

(b) Typical XPS C1s spectrum of a-C film

(c) Optical gap versus $\Delta \mathrm{E}=\mathrm{E}_{\mathrm{Cls}}-\mathrm{E}_{\mathrm{VBM}}$ for diamond, graphite, polyethylene, paraquaterphenyl and polyisoprene (named PE, QP and PI in the graph rspectively).

(d) Optical gap of carbon films derived from $\mathrm{mFB}$ model versus $\Delta \mathrm{E}=\mathrm{E}_{\mathrm{Cls}}-\mathrm{E}_{\mathrm{VBM}}$

(e) Optical gap of carbon films derived from the original FB and from TL models versus $\Delta \mathrm{E}=\mathrm{E}_{\mathrm{C} 1 \mathrm{~s}}-\mathrm{E}_{\mathrm{VBM}}$

Figure 4: (a) Transition energy, $\mathrm{E}_{0}{ }^{\mathrm{TL}}$, from $\mathrm{TL}$ model versus $\mathrm{E}_{\max }{ }^{\mathrm{mFB}}$ from $\mathrm{mFB}$ model for amorphous carbon films

(b) Transition lifetimes from TL versus mFB model

Figure 5: (a) Typical FT-IR spectrum in the $2800-3100 \mathrm{~cm}^{-1}$ wavenumber range ("sym." and "asym." stand respectively for symmetric and asymmetric stretching modes).

(b) $\mathrm{Eg}^{\mathrm{mFB}}$ and $\mathrm{E}_{\max }{ }^{\mathrm{mFB}}$ versus the ratio of FT-IR absorption area between aromatic $\mathrm{C}-\mathrm{H}$ and olefinic $\mathrm{C}-\mathrm{H}_{1,2}$ bonds. 

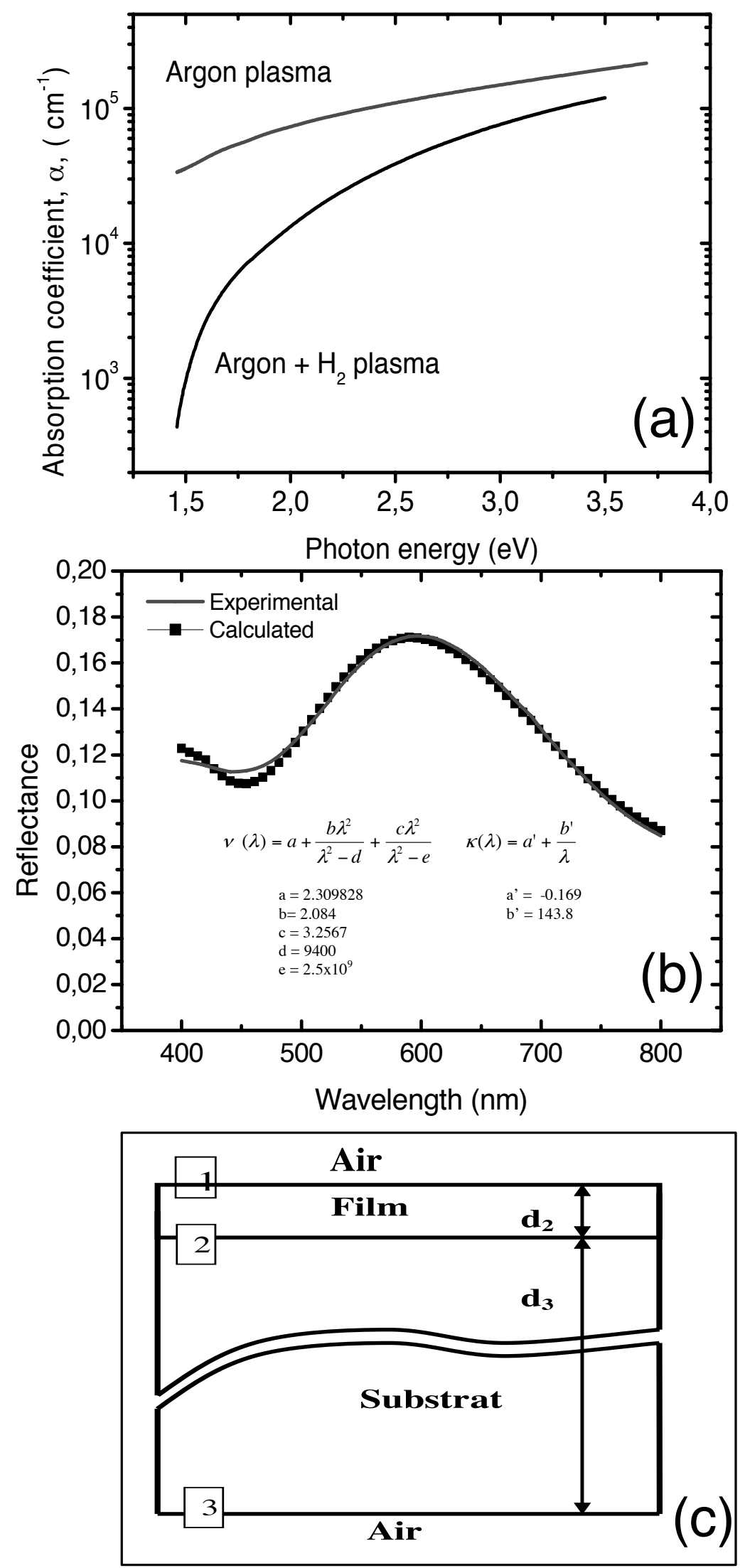


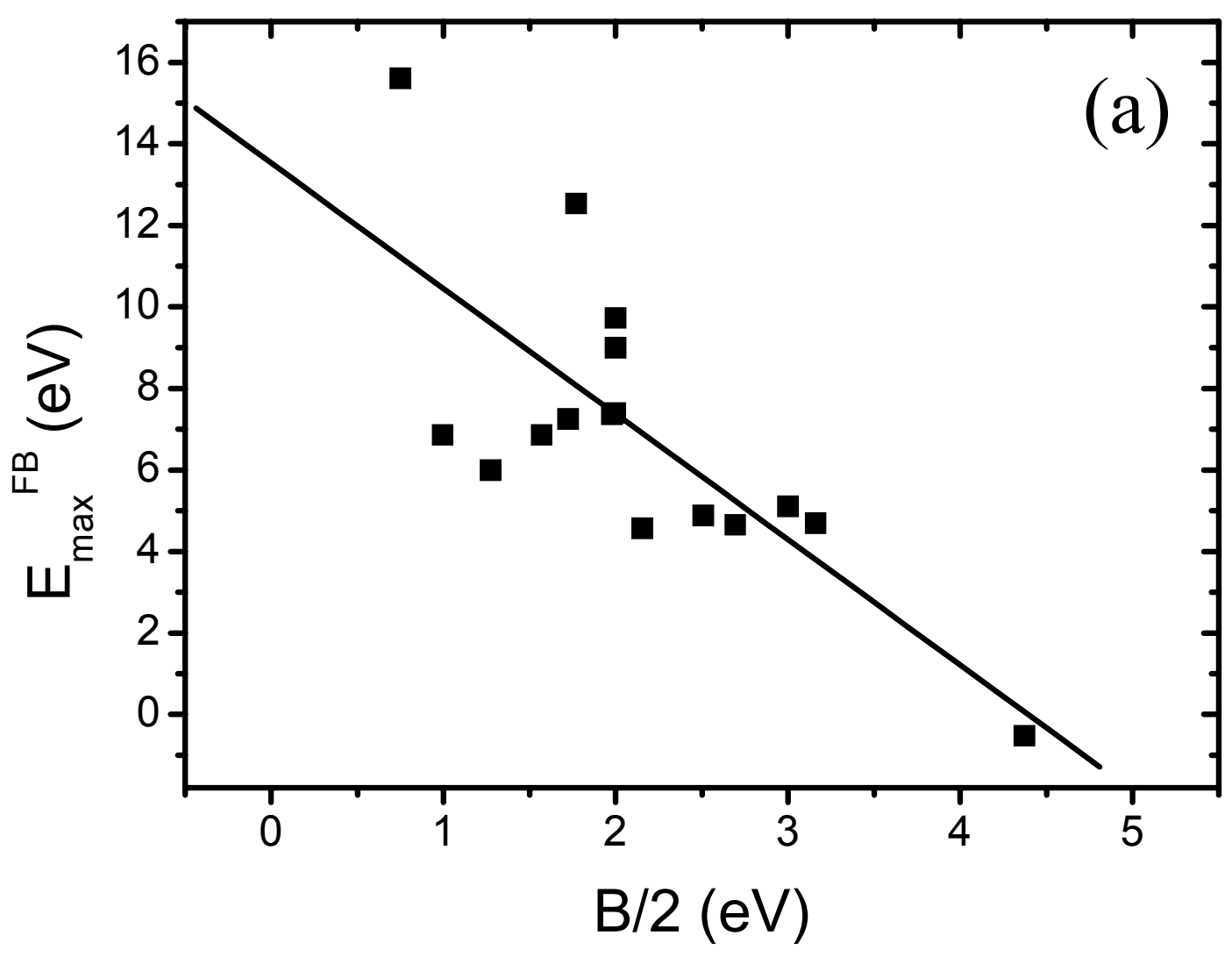




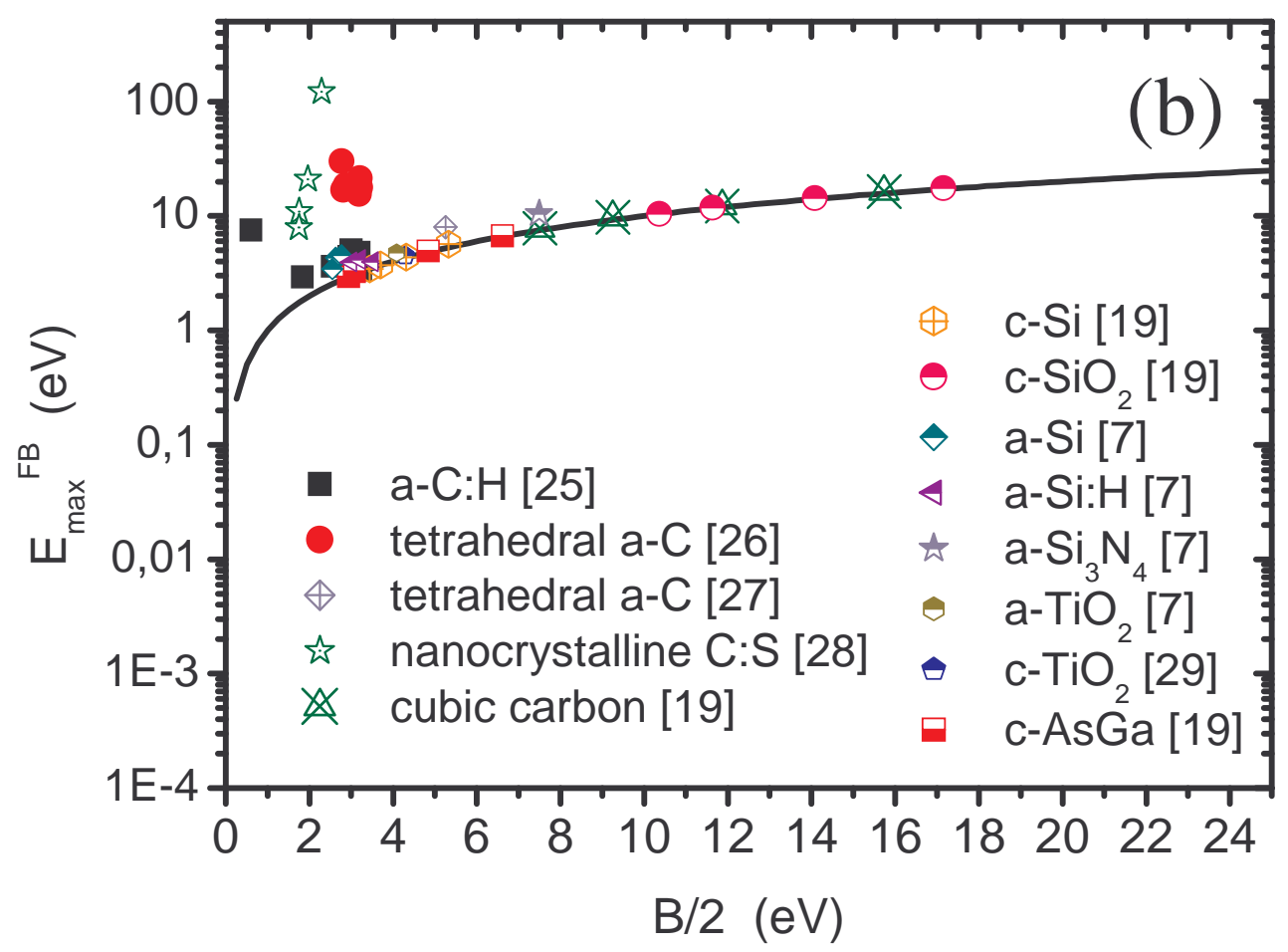




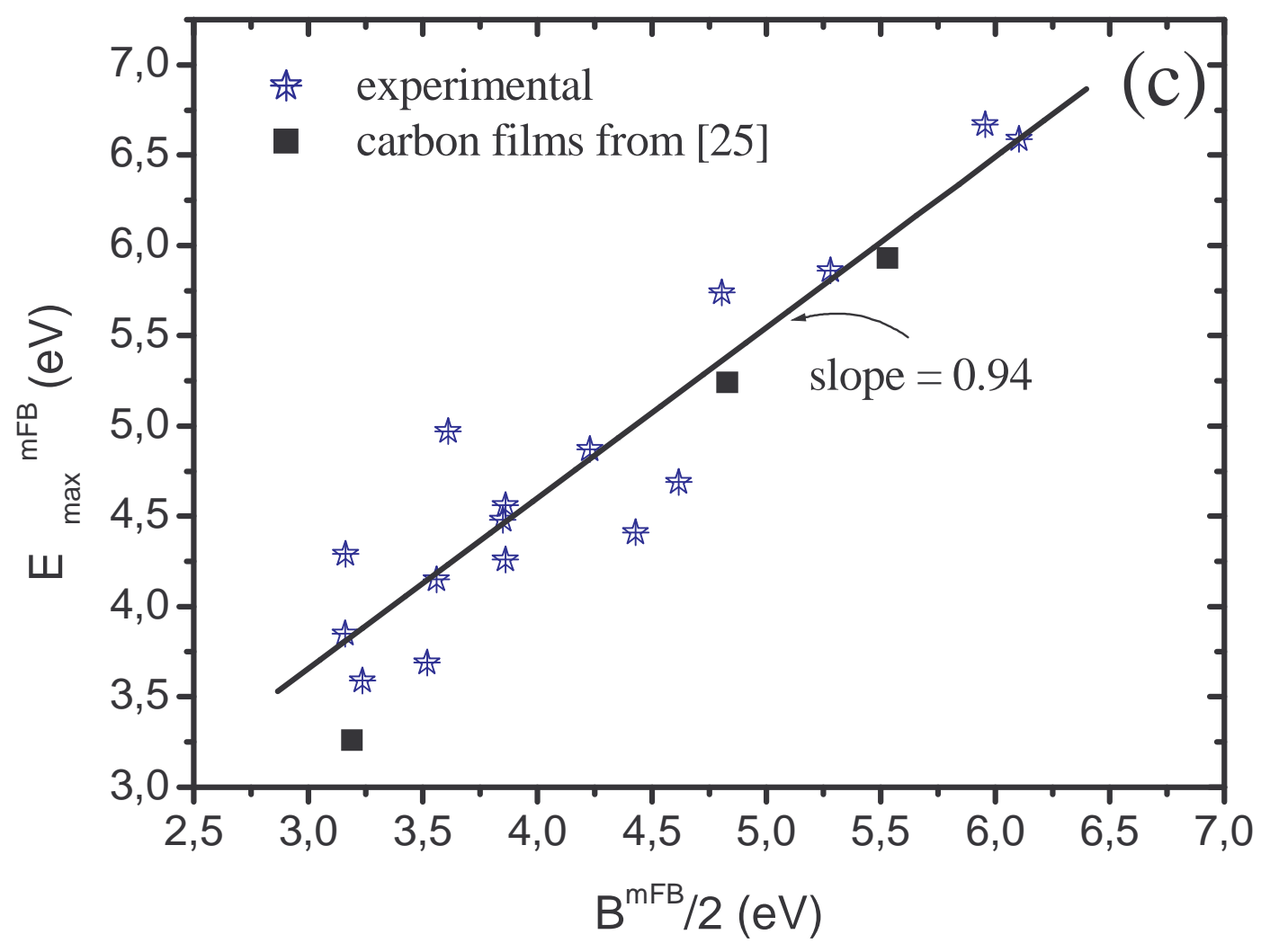



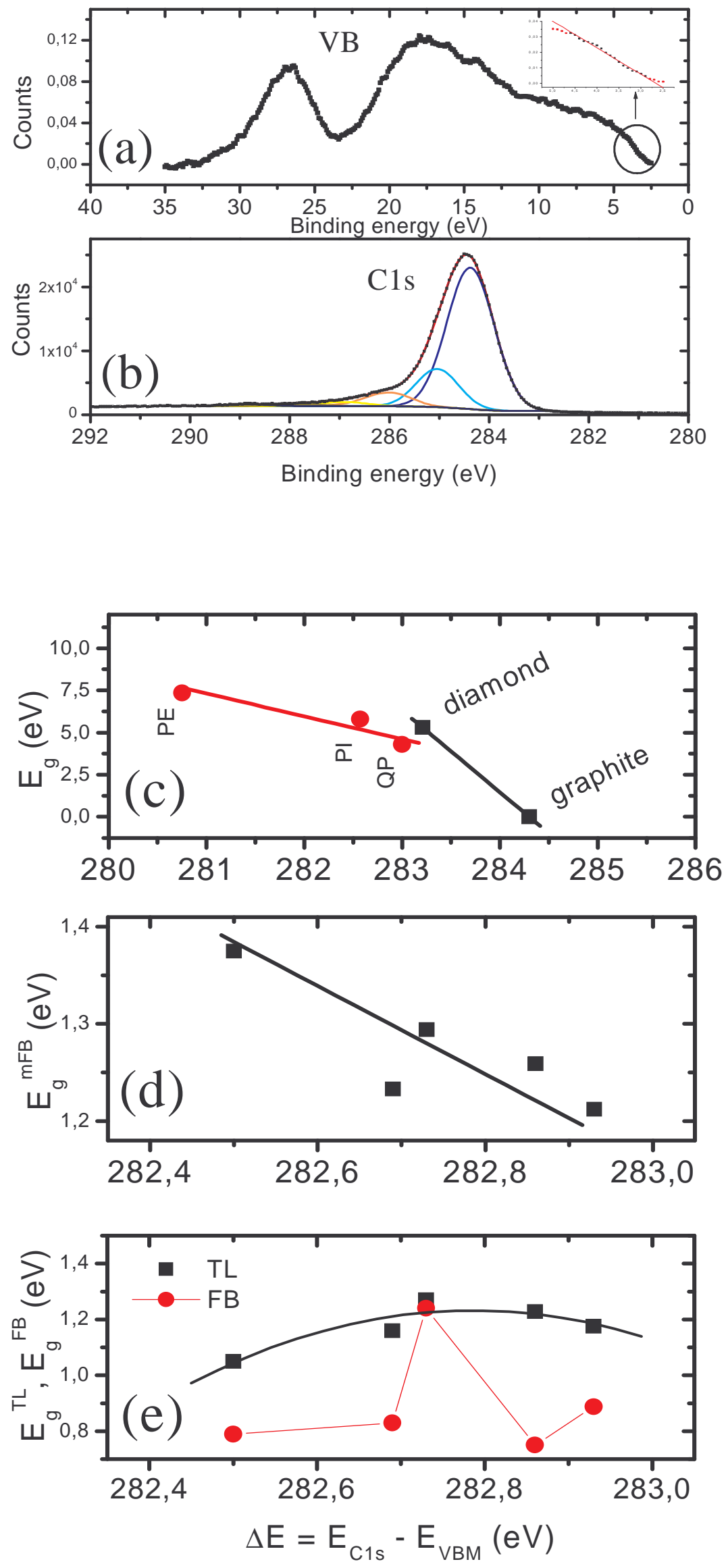

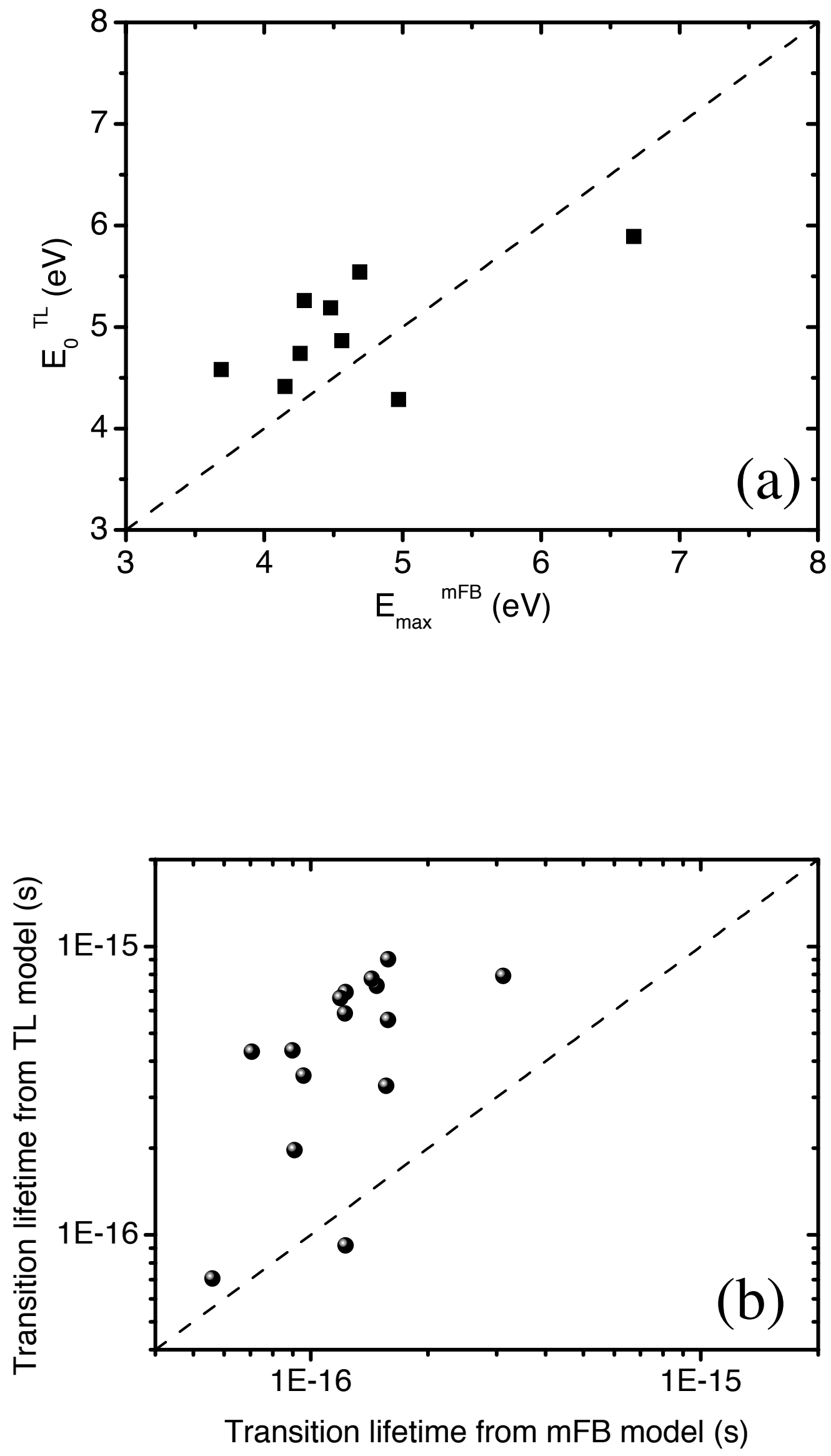

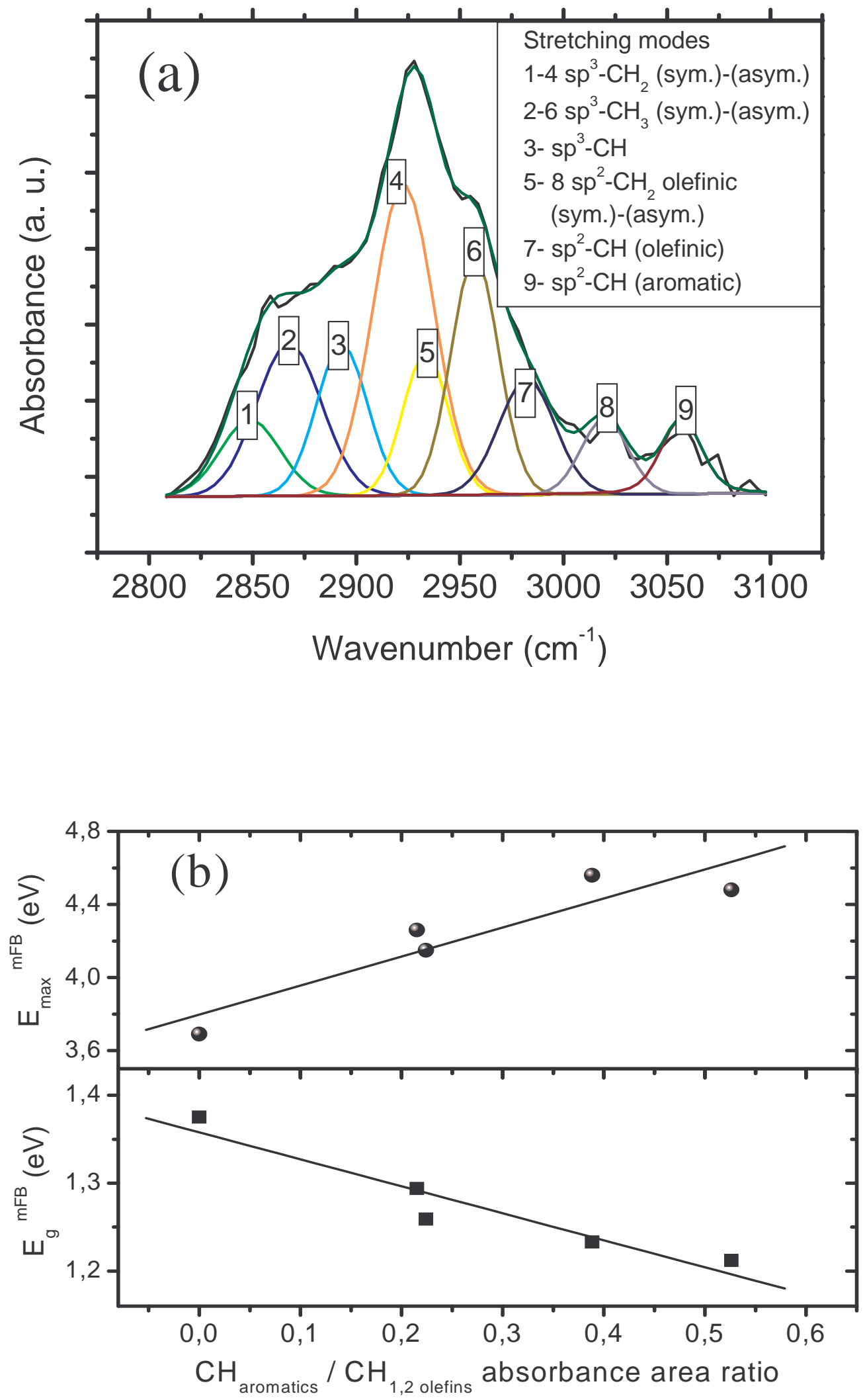